\title{
JESÚS RECHAZA DE PLANO LA PRETENSIÓN DE LOS ONCE DE RESTAURAR EL CÍRCULO DE LOS DOCE APÓSTOLES
}

\author{
Josep Rius-Camps ${ }^{1}$ \\ Ateneu Universitari Sant Pacià. Facultat de Teologia de Catalunya \\ riuscamps@yahoo.es
}

\begin{abstract}
RESUMEN
En la Editio Critica Maior, DIE APOSTELGESCHICHTE (4 vols., Stuttgart 2017) no hay mención en el volumen principal (Teil 1.1) de una variante del Códice Beza, AПOKATACTANEIC EIC (Hch 1,6), que completamente cambia el sentido de la frase, por considerar los editores (Teil 2) que la preposición EIC no sería sino un fenómeno de duplografía. De hecho, la lección de Beza está del todo justificada pues, a diferencia de la pregunta formulada por los apóstoles a Jesús en el texto habitual, en este texto la pregunta era: «Señor, ¿es en este momento cuando vas a restaurar para el reino de Israel...?» Jesús cortó en seco su pregunta, dejando truncada la frase de los Once que habrían intentado preguntar sobre la restauración de las tribus de Israel, ahora que la muerte de Judas había reducido a los apóstoles, a quienes Jesús había designado para que representaran las tribus, a Once. En los 26 versículos de Hechos 1, hay 22 variantes respecto al texto alejandrino que funcionan juntas para explicar la dura respuesta de Jesús y su negativa a reemplazar a Judas como el duodécimo apóstol antes que dejara la tierra. De acuerdo con esto, al final del capítulo, Matías es visto como elegido para existir al lado del original Doce apóstoles, y no, como en el texto alejandrino, como un nuevo y enteramente integrado miembro del grupo.
\end{abstract}

Palabras ClavE: Códice Beza, texto Alejandrino, Editio Critica Maior, restauración, duplografía, Once/Doce apóstoles, frase truncada, votación.

JESUS FLATLY REJECTS THE CLAIM OF THE ELEVEN

TO RESTORE THE CIRCLE OF THE TWELVE APOSTLES

\section{ABSTRACT}

In the Editio Critica Maior, Die Apostelgeschichte (4 vols., Stuttgart 2017) there is no mention in the main volume (Teil 1.1) of a variant reading in Codex Bezae, АПОКАТАCTANEIC EIC (Acts 1.6), which completely changes the meaning of the sentence. The lack of indication is due to the editors' decision (Teil 2) that the preposition EIC was no more than an occurrence of diplography. In fact, the Bezan reading is fully justified because, unlike the question asked by the apostles in the familiar text, in this text the question was: "Lord, is this when you are going to restore to the Kingdom of Israel...?" Jesus cuts short their question, leaving unfinished the question of the Eleven who appear to have intended to ask about the restoration of the tribes to Israel, now that the death of Judas had reduced 
the apostles, whom Jesus had designated as representing the tribes, to Eleven. In the 26 verses of Acts 1, there are some 22 variants with the Alexandrian text, which function together to explain the harsh response of Jesus and his refusal to replace Judas as the twelfth apostle before leaving the earth. Accordingly, at the end of the chapter, Matthias is seen as being elected to exist alongside the original Twelve apostles, and not, as in the Alexandrian text, as a new and fully integrated member of the group.

Keywords: Codex Bezae, Alexandrian text, Editio Critica Maior, restoration, diplography, Eleven/Twelve apostles, sentence cut short, vote.

Hace aproximadamente un año que presenté Novum Testamentum Graecum. Editio Critica Maior de los Hechos de los Apóstoles, recientemente publicada (Deutsche Bibelgesellschaft, Stuttgart 2017), en el Seminari dels Escriptors Prenicens, que dirijo hace ya más de veinticinco años en la Facultat de Teologia de Catalunya. Tratándose de una ECM, deberían encontrarse reseñadas en ella todas las variantes que presentan los manuscritos griegos. Pues bien, justo en los Prolegómenos, pudimos comprobar que no había constancia de una variante del Códice Beza que podría dar un vuelco considerable al sentido de la frase, omisión que, ante nuestra reclamación, los responsables de la edición intentaron minimizar diciendo que se trataba de una duplografía, es decir, de un error de copista, y que ya habían dejado constancia de ello en un volumen aparte ${ }^{2}$ Al insistir nosotros en que no se trataba de un error, sino de una variante importantísima, se excusaron diciendo que mirarían de suplirla en la edición on line. Sin más comentarios.

Para facilitar la comprensión de este pasaje en el Códice Beza, examinaré a continuación su posición en los Prolegómenos, Hch 1,1-26.

\section{BREVE COMPENDIO DEL PRIMER VOLUMEN DE LA DEMOSTRACIÓN A TEÓFILO}

Lucas abre el segundo volumen, también mal llamado Hechos de los Apóstoles, con unos amplios Prolegómenos. En primer lugar brinda a Teófilo un breve resumen del contenido del primer volumen:

${ }^{1}$ Por motivos de salud, no me fue posible enviar mi colaboración para la Miscelánea que la revista ForTVNATAE ha dedicado a mi gran amigo Juan Barreto en el número 30. Una vez ya me he restablecido, me han brindado la posibilidad de colaborar en el próximo número 31 de FORTVNATAE.

${ }^{2}$ Los dos primeros volúmenes presentan el texto de Hch con su consiguiente aparato crítico (el pasaje de Hch 1,6 se encuentra en Parte 1.1, p. 6); un tercer volumen contiene los materiales suplementarios (en concreto, Parte 3, p. 21). 
El primer volumen lo compuse sobre todo aquello, oh Teófilo, que Jesús hizo y enseñó desde el comienzo hasta el día en que fue elevado a lo alto ${ }^{3}$, después de haber dado instrucciones a los apóstoles a quienes había elegido por medio del Espíritu

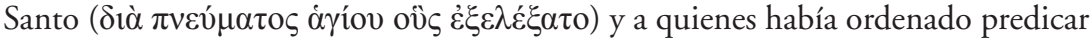
el evangelio ${ }^{4}$.

En el primer volumen de su escrito dirigido a Teófilo Lucas había dejado constancia de la elección de los Doce, con sus nombres y sobrenombres o apodos, coordinados todos ellos mediante la conjunción "y»", para que representaran en plano de igualdad a las doce tribus de Israel (Lc 6,13-16); más adelante, había reseñado la convocatoria de los Doce y su envío para que proclamaran el reino de Dios y curaran a los más débiles $(9,1-2)$. Ahora, en los Prolegómenos del segundo volumen, resume los dos momentos poniendo énfasis en primer lugar en que Jesús había llevado a cabo la elección de "los apóstoles» (y ya no de "los Doce», pues su función había quedado desactivada con la defección de Judas) por inspiración del Espíritu Santo, «a quienes había elegido por medio del Espíritu Santo» ${ }^{6}$, y añadiendo un nuevo detalle a la instrucción que Jesús había impartido a los Doce en el momento de su elección, "y a quienes había ordenado predicar el evangelio», un detalle que solo se ha conservado en el Códice Beza y, en parte, en algunas antiguas versiones siríacas y $\operatorname{coptas}^{7}$, si bien Lucas ya lo había insinuado en el primer volumen cuando dijo que "Jesús los envió a predicar el reino de Dios» $(\operatorname{Lc} 9,2)^{8}$.

Lucas, que en nuestra opinión sigue a Juan, había puesto de relieve el fracaso total de la misión de Jesús como Mesías de Israel en la inscripción escrita sobre él en la cruz en caracteres griegos, romanos y hebreos: EL REY DE LOS JUDÍOS ES ESTE (Lc 23,38 D pm lat; Jn 19,19-20) y había conferido mucho resalte a la acción de Jesús

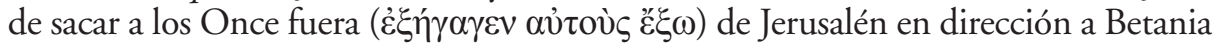
(Lc 24,50 D pm lat), lo mismo que había hecho Yahveh cuando sacó afuera a Abraán

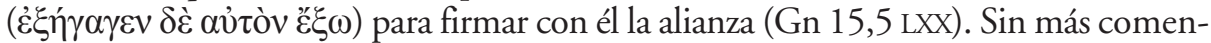
tarios" ${ }^{9}$ constataba que se había apartado de ellos (Lc 24,50-51). A renglón seguido,

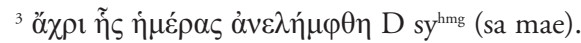

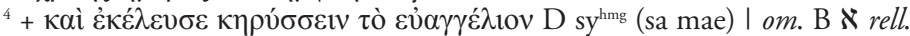

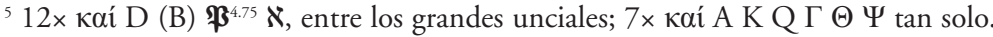

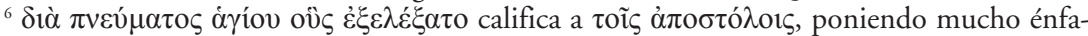
sis en esta nueva información al anticipar la mediación del Espíritu Santo en la elección de los após-

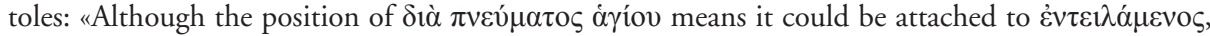
'having instructed', the phrase in fact belongs to oṽ $\dot{\varepsilon}^{\xi} \xi \varepsilon \lambda \hat{\varepsilon} \xi \alpha \tau o$, 'whom he chose'. It is unusual for a relative to be placed after a qualifying phrase but other instances of such a construction occur in Luke's writings: e.g. Lk. 24.7; Acts 1.10; 3.19; 4.33; 5.13; 7.35; 9.14; 12.25; 16.14; 19.4, 20» (RiusCamps and Jenny Read-Heimerdinger, The Bezan Text of Acts in Codex Bezae, I, p. 62, n. 14).

${ }^{7} \mathrm{D}^{\mathrm{h}} \mathrm{y}^{\mathrm{hmg}}$ (sa mae).

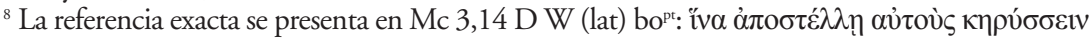

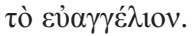

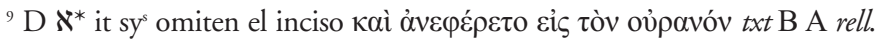


sin embargo, pone fin al primer volumen comprobando que los Once «regresaron

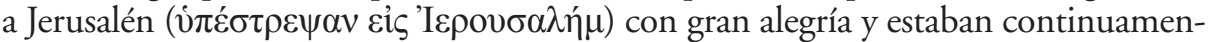
te en el Templo alabando a Dios» (24,52-53, recordado por Lucas a Teófilo en el segundo volumen: Hch 1,12). Los Once siguen plenamente identificados con la institución judía ("Jerusalén", en sentido sacral) ${ }^{10}$ y con el Templo, haciendo caso omiso a la última acción de Jesús de «sacarlos fuera» de Jerusalén.

\section{CONFABULACIÓN DE LOS ONCE EN EL MONTE DE LOS OLIVOS PARA PLANTEAR A JESÚS LA CUESTIÓN CRUCIAL}

Después que Jesús hubiese compartido con ellos, cuarenta días después de resucitar, la última comida en el Monte de los Olivos, ordenándoles predicar el Evangelio,

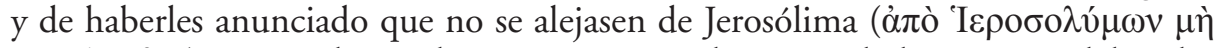
$\chi \omega \rho$ í $\zeta \varepsilon \sigma \theta \alpha \imath)$ y aguardasen el inminente cumplimiento de la promesa del Padre (no después de la fiesta de Pentecostés, según puntualiza el Códice Beza), los Once se reunieron aparte para deliberar sobre su situación antes de que Jesús se apartara de ellos definitivamente:

Ellos (los Once), pues, que se habían reunido aparte, se pusieron a interpelarlo

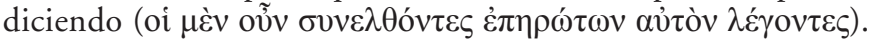

Se trata de una interpelación en toda regla, en un tono muy solemne ${ }^{11}$, y no de una simple pregunta, según consta en el texto alejandrino ${ }^{12}$.

La pregunta que formulan los Once a Jesús es diáfana, si seguimos el texto alejandrino:

Señor, ¿es en este preciso momento cuando vas a restaurar el reino para Israel ( $\tau \grave{v} v$

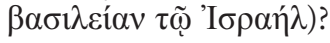

Jesús habría dejado que los Once terminasen de formular la pregunta. Tanto es así que en $\mathrm{NA}^{28}$ ni siquiera han considerado reseñables las dos variantes que leemos en el Códice Beza:

${ }^{10}$ "When Luke refers (or has a character refer) to the town as a geographical place, devoid

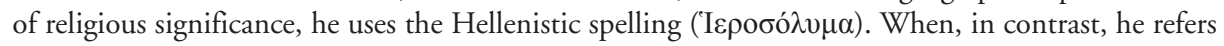
(or has a character refer) to the city as the seat of Jewish authority, the centre of the Jewish religion,

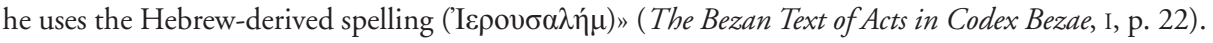

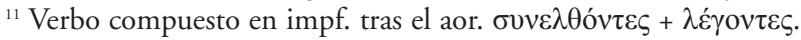

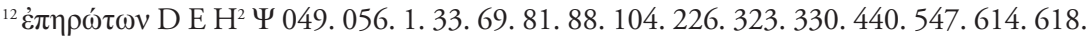
927. 945. 1241. 1243. 1245. 1270. 1505. 1611. 1646. 1739. 1828. 1837. 1854. 1891. 2147. 2412. 2492. 2495 | ท่ 
Señor, ¿es en este preciso momento cuando vas a restaurar ${ }^{13}$ para el reino de Israel

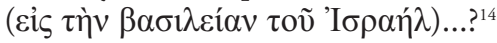

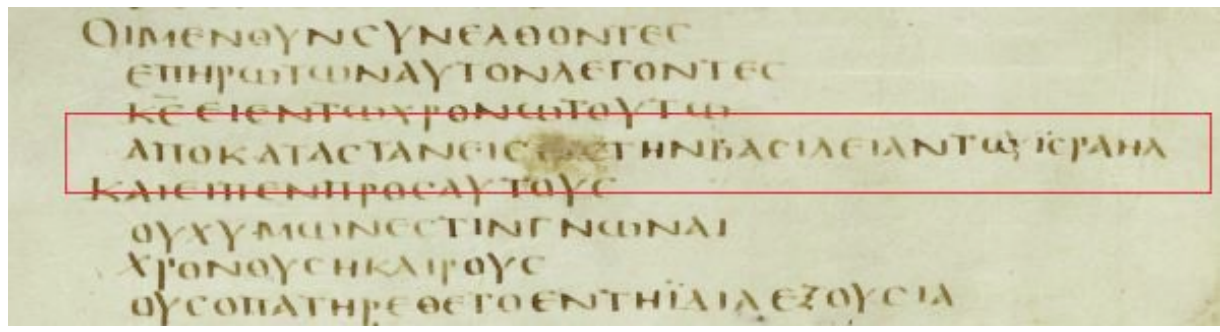

Como se puede observar fácilmente sobre el manuscrito on line (supra), un corrector posterior del Códice Beza, identificado com $\mathrm{D}^{\mathrm{H}}$ (entre 450-500), ha

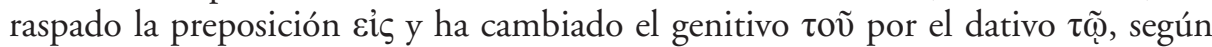
las Adnotationes editoris de F. H. Scrivener ${ }^{15}$. Así pues, según la versión original del Códice Beza, Jesús habría dejado deliberadamente truncada la interpelación de los Once, indicándolo mediante la conjunción $\kappa \alpha i ́$, en lugar del $\delta \varepsilon ́$ esperado para introducir una respuesta a una pregunta, por estar en absoluto desacuerdo con una futura restauración del círculo de los Doce. Lucas utiliza con frecuencia este artificio en el segundo volumen, ya sea interrumpiendo un dicho, ya sea aplazando un discurso en un momento crucial: Hch 4,1 (interrupción) ${ }^{16} ; 10,19$ (interrupción) ${ }^{17}$; 10,44 (interrupción) ${ }^{18} ; 11,15$ (interrupción) ${ }^{19} ; 13,2$ (interrupción) ${ }^{20} ; 17,32$ (aplazamiento) $)^{21} ; 20,9$ (interrupción) ${ }^{22} ; 22,22$ (aplazamiento) ${ }^{23} ; 24,22$ (aplazamiento) ${ }^{24}$;

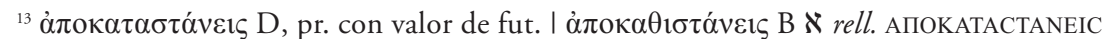

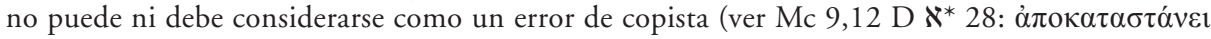
$\pi \alpha ́ v \tau \alpha)$ como presupone la ECM III: «05(*f2, C1f1)». Véase Bauer-Aland, Wörterbuch ${ }^{6}$, p. 183: «NF

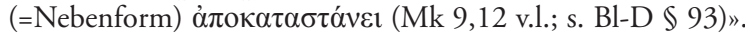

${ }^{14} \mathrm{D}^{*}$ 61. 326. 365. 522. 1611. 1837 eth; SevGab. La prep. EIC D*, ha sido raspada por un corrector posterior a $\mathrm{D}$ (ca. 400: $\left.\mathrm{D}^{\mathrm{H}} 450-500\right)$ por presunta duplografía con la sílaba final de AПОКАТАCTANEIC, ECM I, p. 6: «287 a $\alpha \pi 0 \kappa \alpha \theta 1 \sigma \tau \alpha v \varepsilon 1 \zeta . . .05\left({ }^{*} \mathrm{f} 2, \mathrm{C} 1 \mathrm{f1}\right) »$, a saber, de acuerdo con la Lista de los

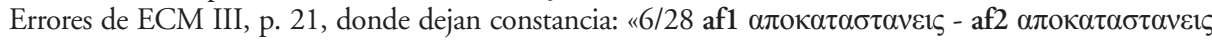

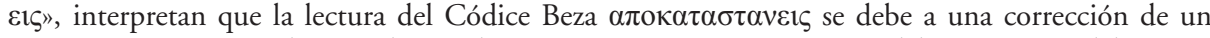
copista $(\mathrm{C} 1)$, pues en el original $\left({ }^{*}\right)$ se leía $\alpha \pi$ \% $\alpha \theta 1 \sigma \tau \alpha v \varepsilon 1 \zeta$, y que $\varepsilon 1 \zeta$ se debe a un error del copista del Códice Beza por duplografía con $\alpha \pi$

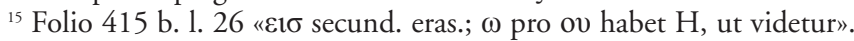

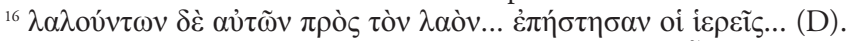

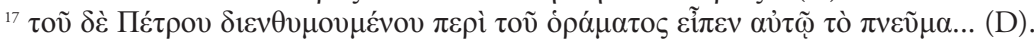

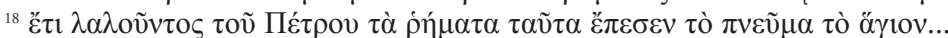

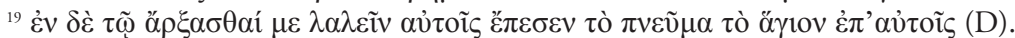

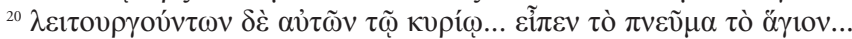

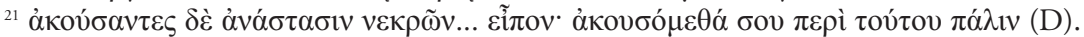

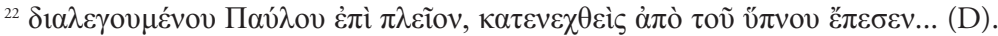

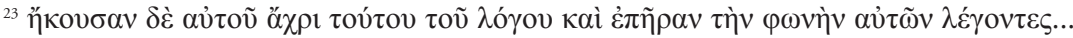

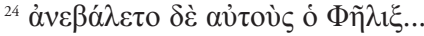


24,25 (aplazamiento) ${ }^{25} ; 26,24$ (aplazamiento) ${ }^{26}$. En nuestra escritura, ya muy evolucionada, la manera de reflejar una frase truncada se hace sirviéndose de los puntos suspensivos.

La importancia de esta serie de variantes es capital, pues está en juego si Jesús aceptó en primera instancia el contenido de la pregunta que le formularon los Once o bien si la rechazó de plano. He dicho en primera instancia, puesto que la respuesta consiguiente de Jesús no representa una salida por la tangente, sino un cambio radical de sus planes. En caso que les hubiese dejado terminar la pregunta, podría interpretarse que Jesús resucitado no había renunciado a restaurar el reino para Israel, como una restauración temporal de la realeza davídica, aun cuando no lo haya restaurado en este momento. Por el contrario, en caso que la haya rechazado de plano, dejando truncada la interpelación de los Once, la restauración que harán a precario más adelante los Once carecería por completo de legitimación.

\section{JESÚS RECHAZA DE PLANO LA RESTAURACIÓN DE LOS DOCE APÓSTOLES}

Jesús no solo ha dejado truncada la interpelación que le habían dirigido los Once, sino que con un kaí inusual para introducir una respuesta, como si se tratara de dos líneas de pensamiento (de los apóstoles y de Jesús) que corren paralelas, el Códice Beza enlaza la sentencia que había quedado truncada con la advertencia de Jesús para que desistan de preguntar:

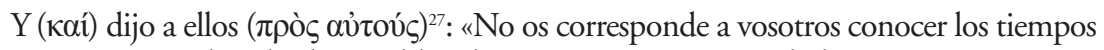
o épocas que el Padre ha establecido con su propia autoridad $»^{28}$.

\section{EN SU LUGAR PROPONE A LOS ONCE UN PROYECTO UNIVERSAL}

El plan A que Dios había ofrecido en los comienzos a las setenta naciones, pero que tan solo Israel aceptó, se ha venido abajo tras el rechazo del Mesías por parte

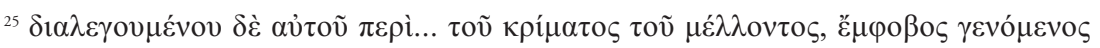
ó $\Phi \tilde{\eta} \lambda \lambda_{1} \xi \dot{\alpha} \pi \varepsilon \kappa \rho i ́ \theta \eta \ldots$

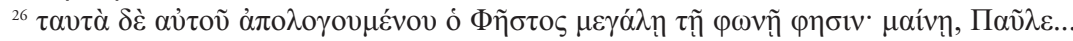

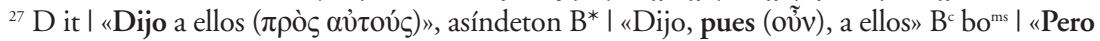

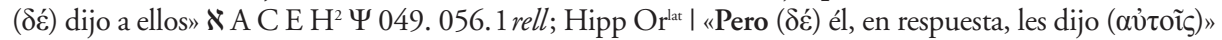
E 1884. «The conjunction אaí in D05 shows that Jesus' response is viewed neither as the correlative of the $\mu \dot{\varepsilon} v$ oũv clause nor as a new development in answer to the apostles' question» (The Bezan Text of Acts in Codex Bezae, I, p. 253).

${ }^{28}$ Hch 1,7 B D rell.
} 
de los sumos sacerdotes (Jn 18,35; 19,6-8.15-16). En el Gólgota ha quedado grabado para siempre el rechazo por parte de Israel del proyecto que Dios tanto había mimado (19,19-22). Jesús, y con él Dios, ha cambiado de planes. El plan B es un plan universal, sobretodo según la versión del Códice Beza:

«Al contrario ( $(\dot{\alpha} \lambda \lambda \dot{\alpha})$, recibiréis fuerza, cuando el Santo Espíritu venga sobre vosotros, y seréis testigos de mí no solo en (

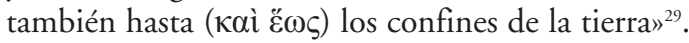

Jesús ha dejado definitivamente de lado el proyecto que le había sido encomendado de ser el Mesías de Israel, después de su estrepitoso fracaso, y, tras la defección de Judas y con ello la pérdida de la representatividad que había conferido a los Doce, propone ahora a los Once un proyecto universal, un proyecto que deben ofrecer, sin pasar ya por Israel, a todas las naciones de la tierra.

\section{LOS ONCE AÚN ESPERABAN QUE, SI VEÍAN A JESÚS MIENTRAS SUBÍA AL CIELO, ESTE LES DEJARÍA EN HERENCIA EL ESPÍRITU PROMETIDO, COMO HIZO ELÍAS CON ELISEO}

Lucas narra dos veces sucintamente la ascensión de Jesús al cielo. La primera vez, al final del primer volumen, como conclusión de un día simbólico que abrazaba todos los relatos de las apariciones de Jesús:

Y sucedió que, mientras él los bendecía, se apartó I separó de ellos ${ }^{30}$.

Beza, Sinaítico original, las antiguas versiones latinas y siríacas ( $D \boldsymbol{N}^{*}$ it sys) no precisan su paradero; en cambio, la mayoría de manuscritos añaden: «e iba siendo

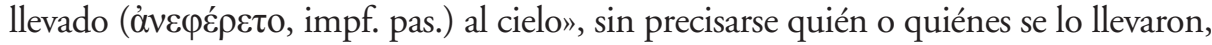
obviamente serían los ángeles. Lo enrevesado de esta construcción deja entrever que se trata de una glosa.

Al principio del segundo volumen, al término de un período de cuarenta días, Lucas precisa algo más a Teófilo:

${ }^{29} \mathrm{D} \mathrm{A} \mathrm{C}^{*}$ 81. 226c. 323. 547*. 945. 1243. 1611 I txt $\mathrm{B} \times \mathrm{C}^{3} \mathrm{E} \mathrm{H}^{2} \Psi$ 049. 056. 1. 33. 69. 88. 104. 226*. 330. 440. 547`. 614. 618.927. 1241. 1245. 1270. 1505. 1646. 1739s. 1828. 1837. 1854.

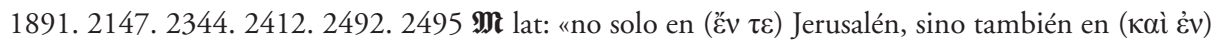

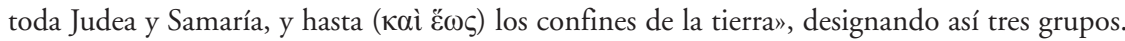

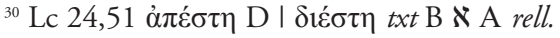




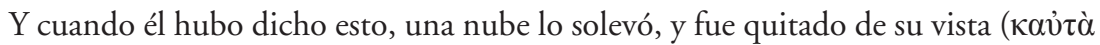

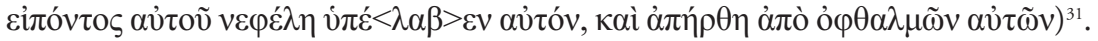

En cambio, en la mayoría de manuscritos se observa un orden distinto de las palabras que modifica notablemente la frase:

Y habiendo dicho esto, mientras ellos miraban, fue llevado hacia arriba (غ̇лท́ $\rho \theta \eta)$,

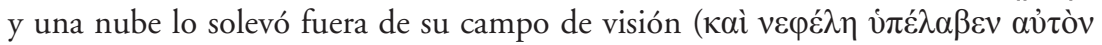

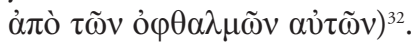

Se observa de nuevo la tendencia a magnificar la ascensión, con perjuicio del sentido original de la «nube», símbolo de la presencia de Dios, que lo habría levantado empujando de abajo arriba para quitarlo del alcance de su mirada. Los Once se han quedado con la vista fijada en el cielo esperando que Jesús les dejaría en herencia el Espíritu Santo que les había prometido, como hizo Elías con Eliseo cuando este logró verlo mientras era arrebatado al cielo y le dejó en herencia su manto (2Re [4Re LXX] 2,2-13):

Mientras miraban fijamente al cielo al tiempo que se iba, he aquí que dos varones se habían presentado a su lado en vestidura blanca ${ }^{33}$. Finalmente, ellos dijeron: «¡Galileos!, ¿por qué estáis en pie mirando fijamente al cielo? El mismo Jesús que ha sido elevado a lo alto de entre vosotros, así vendrá del mismo modo como lo habéis visto irse hacia el cielo» ${ }^{34}$.

Por tercera vez Lucas hace aparecer en escena a estos «dos varones»: son Moisés y Elías, según explicitó la primera vez (Lc 9,30-33; cf. 24,4) ${ }^{35}$. Los discípulos se han quedado mirando fijamente al cielo mientras Jesús se alejaba de ellos. Moisés y Elías les instruyen para que dejen de mirar al cielo y descubran su presencia en la vida cotidiana.

${ }^{31}$ Hch 1,9 D (69).

${ }^{32}$ Hch 1,9 txt $\mathrm{B}$ « A rell.

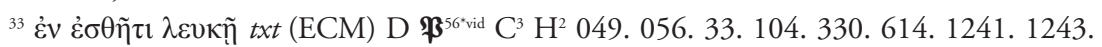
1245. 1270. 1505. 1611. 1739. 1828. 1837. 1854. 2147. 2344. 2412. 2492. $2495 \mathfrak{M}$ gig sy I غ̇v

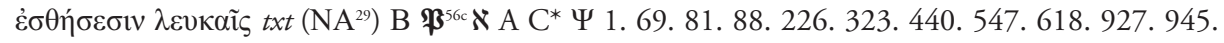
1175 lat; Eus.

${ }^{34}$ Hch $1,10-11$.

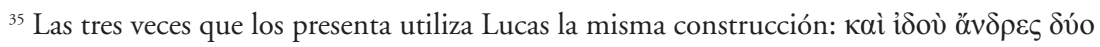
(Lc 9,30; 24,4 y Hch 1,10); sólo en la primera aparición explicita el nombre de esos dos personajes:

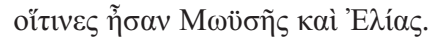




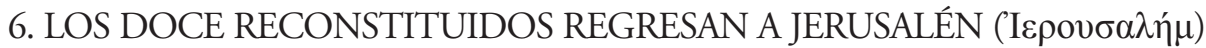

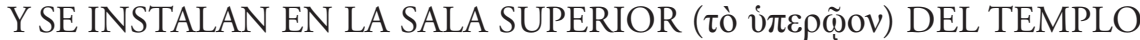

En vez de regresar a Jerosólima y permanecer allí inactivos, como Jesús les

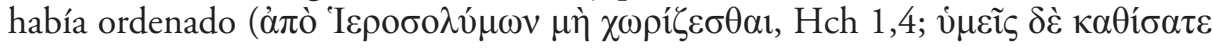
$\dot{\varepsilon} v \tau \tilde{n} \pi$ ó $\lambda \varepsilon 1$, Lc 24,49), Lucas remarca de nuevo:

Entonces regresaron a Jerusalén ( conocido como El Olivar, que está cerca de Jerusalén, un viaje de día de sábbath. Cuando entró é $\mathrm{l}^{36}$, subieron a la sala superior, donde permanecían esperando ${ }^{37}$.

Si hubiesen regresado a Jerosólima, a la ciudad sin más calificativos, y hubiesen permanecido allí esperando la realización de la Promesa, habrían cumplido con creces la doble orden que Jesús les había impartido. Pero ellos han regresado a Jerusalén, en sentido sacral, quedando así plenamente identificados con la institución del Templo. A pesar de que no era un día de sábbath cuando regresan a Jerusalén, mediante la referencia a la distancia que se permite a un judío caminar en un sábbath, a saber, la distancia que mediaba entre el tabernáculo del desierto, situado en el centro del campamento, y los límites exteriores del mismo, Lucas recuerda a Teófilo que los apóstoles han vuelto al centro del culto, de la Torá y de la autoridad judía, como si nada hubiese ocurrido. El verbo en singular ( $\varepsilon i \delta \tilde{\eta} \lambda \theta \varepsilon v)$ y el artículo anafórico

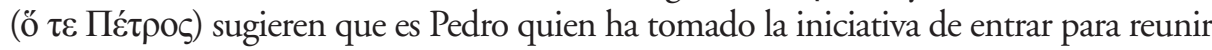
a los otros diez, nombrados por su nombre, en la sala superior, una de les dependencias del Templo ${ }^{38}$, bien conocida de Teófilo ${ }^{39}$. Servían de lugares de reunión, salas de estudio y lugares de oración para los escribas. Sin embargo, cuando llegan allí los Once, la sala superior del Templo ya estaba ocupada por los hermanos de Jesús:

Eran Pedro y Juan, Santiago y Andrés, Felipe y Tomás, Bartolomé y Mateo, Santiago el hijo de Alfeo, Simón el Zelota y Judas, hijo de Santiago. Todos estos perseveraban unánimemente en la oración, con sus mujeres e hijos, además de María, la madre de Jesús, y sus hermanos ${ }^{40}$.

${ }^{36} \varepsilon \dot{\epsilon} \sigma \tilde{\eta} \lambda \theta \varepsilon v \mathrm{D}$ । $\operatorname{\varepsilon i\sigma } \tilde{\eta} \lambda \theta$ ov txt B $\boldsymbol{N}$ rell.

${ }^{37}$ Hch $1,13 \mathrm{a}$.

${ }^{38}$ The Message of Acts I, p. 101, n. 88. Además de la cita de 1Cr 28,11.20, se podrían añadir cinco menciones más donde vं $\pi \varepsilon \rho \tilde{\omega} o v$ aparece describiendo partes del Templo: 4Re 23,11; 2Cr 3,9 (describe el Sancta Sanctorum); Jr 20,2; Ez 41,7 y 42,5.

${ }^{39}$ A medida que avanzamos en el comentario de los Hechos, Jenny Read-Heimerdinger y un servidor llegamos a la conclusión que el personaje a quien Lucas dedicó su doble obra era nada menos que el hijo de Anás, de nombre Teófilo, que fue sumo sacerdote los años 29-31 de nuestra era,

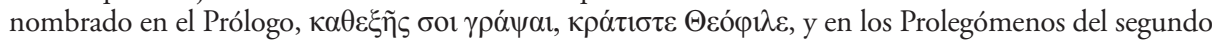

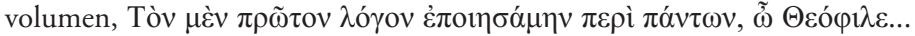

${ }^{40}$ Hch 1,13b-14 D. 
Comparten la estancia dos grupos bien diferenciados, los Once, con sus mujeres e hijos (Códice Beza) ${ }^{41}$, y María, madre de Jesús, y sus hermanos. Los primeros han perdido su representatividad ante Israel y no han logrado recuperarla, pero tienen una información privilegiada, que no tienen los hermanos de Jesús, sobre la ya inminente realización de la Promesa («no después de Pentecostés», 1,5 D). La presencia de los hermanos de Jesús, en un momento tan crítico en que estaba en juego la realización de la Promesa, forzará a Pedro a actuar -como se verá a continuación- contraviniendo la orden de Jesús de permanecer inactivos (cf. Lc 24,49). A pesar de que los hermanos de Jesús nunca habían seguido sus trazas, han hecho valer ahora sus derechos de sangre con el Mesías Jesús. Las mujeres de los Once y María, la madre de Jesús, se interponen entre los dos grupos, pero la tensión entre los dos grupos va in crescendo a medida que se agotan las horas del plazo que Jesús había fijado a los Once.

\section{PEDRO PROPONE RESTAURAR LA FUNCIÓN QUE EJERCÍA JUDAS, PERO DEJANDO VACANTE SU ESCAÑO}

Nadie se mueve en esta Sala superior previamente ocupada por los hermanos de Jesús. Pedro es muy consciente que, si viene el Espíritu Santo sobre todos ellos, los hermanos de Jesús harán valer sus derechos dinásticos. Para salir del atolladero, opta por una solución política: respetar la voluntad de Jesús dejando vacante el escaño que Judas ocupaba y restaurar la función que este había ejercido.

Fue en estos días cuando Pedro se puso en pie en medio de los discípulos ${ }^{42}$ y dijo (pues era el número de personas reunidas con un propósito común metafóricamente ${ }^{43}$ Ciento veinte): «Hermanos, es necesario $(\delta \varepsilon \tilde{\varepsilon})^{44}$ que se cumpla esta escritura

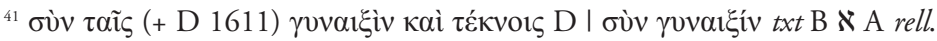

${ }^{42} \mu \alpha \theta \eta \tau \tilde{\omega} \nu \mathrm{D} \mathrm{C}^{3} \mathrm{E} \mathrm{H}^{2} \Psi$ 049. 056. 1. 81.226. 323.330. 440. 547. 614. 618. 927*. 1241. 1243.
}

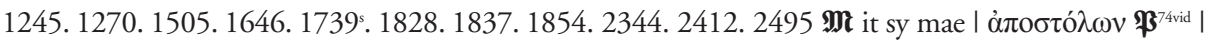
$\alpha \dot{\alpha} \delta \varepsilon \varphi \tilde{\omega} \nu$ txt B $\aleph \mathrm{A} \mathrm{C}^{*} 33.88 .104 .547 .927^{\circ} .945 .1175$ vg sa bo.

${ }^{43}$ Transcribo aquí la nota que pusimos en nuestro Comentario a Hechos en su traducción

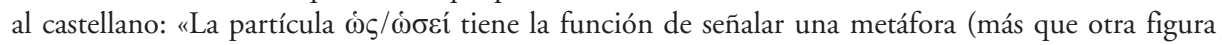

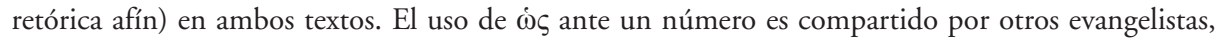
pero el uso de $\mathfrak{\omega} \sigma \varepsilon i$ ante un número se encuentra solamente en Lucas. En Hechos, las dos partículas constituyen un ejemplo de la dualidad de vocabulario que Lucas utiliza deliberadamente para comu-

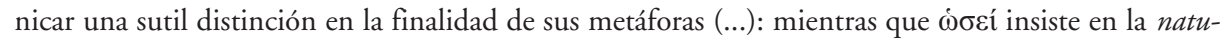
raleza metafórica de la comparación, ஸ́ৎ llama la atención sobre el origen de la metáfora y el punto de comparación. La metáfora en cuestión es el número 120, que representa a Israel en su totalidad» (Josep Rius-Camps y Jenny Read-Heimerdinger, El mensaje de los Hechos de los Apóstoles en el Códice Beza, Estella (Verbo Divino), vol. I, p. 106).

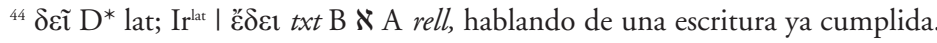


( niente a Judas que actuó como guía de quienes apresaron a Jesús, porque él había sido enumerado entre nosotros, a quien había sido asignada una porción de este ministerio $^{46}$.

Entre el anuncio de la Escritura, cuyo cumplimiento va a aducir, y la cita del libro de los Salmos de David, Lucas ha intercalado un amplio paréntesis, para poner de relieve un hecho que ha alterado por completo los planes iniciales de Jesús, el suicidio de Judas:

A propósito, este lo que hizo fue comprar un campo con la recompensa de su injusticia, y cayó de bruces y se reventó por la mitad y todas sus tripas se desparramaron, un incidente que llegó a ser conocido a todos los habitantes de Jerusalén, de modo que aquel campo fue llamado en su propia lengua Haqueldaimakh, esto es, 'Campo de Sangre' ${ }^{47}$.

La mala muerte de Judas, el castigo de un impío (cf. Sb 4,9 LXX), muy enfatizada en el seno de este inciso parentético, había privado a los apóstoles de la representatividad que Jesús les había otorgado cuando los eligió (cf. Lc 6,13) y a quienes había conferido realeza en la última cena para que, con la llegada del reino de Dios, se sentaran sobre doce tronos como jueces de las doce tribus de Israel (cf. Lc 22,29-30). Ante la nueva situación creada por la presencia de los hermanos de Jesús, los Once

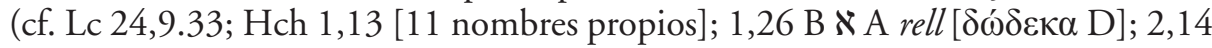
$\mathrm{B} \aleph \mathrm{A}$ rell $\left.\left[\delta \varepsilon \dot{\kappa} \kappa \alpha \mathrm{D}^{*} 1243\right]\right)$, ya no pueden hacer prevalecer sus derechos de representar a Israel.

Pues está escrito en el libro de los Salmos: «Que su finca (ं desierta ("̌ $\rho \eta \mu \mathrm{s})$ y no haya nadie que habite en ella» (Sal 68 [69],26 LXX) y "Que

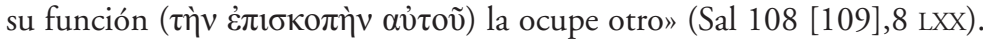

Pedro se ha visto precisado a avalar con una cita escriturística, «es necesario que se cumpla esta ( $\tau \alpha u ́ \tau \eta v)$ Escritura», la propuesta que estaba a punto de presentar a la asamblea, de otro modo ésta la habría rechazado. Por un lado, propone que se debe respetar la voluntad de Jesús, dejando vacío su escaño; por otro, pretende soslayar las pretensiones de los hermanos de Jesús, disponiendo que otro ocupe su función, "para ocupar el lugar de este ministerio y apostolado ( $\tau$ òv $\tau o ́ \pi o v ~ \tau \tilde{\jmath} \varsigma$

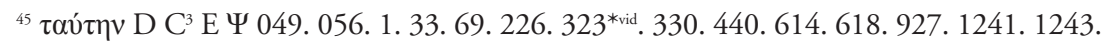
1245. 1270. 1505. 1611. 1646. 1739s. 1828. 1837. 1854.2147.2344. 2412. 2492. $2495 \mathfrak{M l}^{\text {it }}$ vg $^{\mathrm{ms}} \mathrm{sy}^{\mathrm{h}}$; Irlat I om. txt $\mathrm{B} \times \mathrm{A} \mathrm{C}^{*} \mathrm{H}^{2}$ 81. 104. 323 . 945.1175 vg syp; Eus.

${ }^{46}$ Hch $1,16-17$.

${ }^{47}$ Hch 1,18-19. 


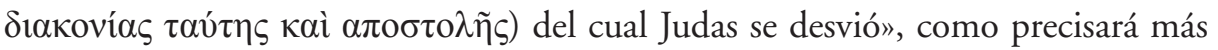
adelante (cf. 1,25).

El futuro candidato, para ocupar la función que Judas había dejado vacante, debía ser discípulo de Jesús de primera hora y testigo de su resurrección:

Es necesario $(\delta \varepsilon \tilde{i})$, por consiguiente, de entre los hombres que nos acompañaron a lo largo del período de tiempo durante el cual entró y salió el Señor Jesús Mesías de entre nosotros, comenzando desde el bautismo de Juan hasta el día en que fue elevado a lo alto de entre nosotros, que en testigo de su resurrección junto con nosotros se convierta uno de estos ${ }^{48}$. datos (dos):

Según el Códice Beza, es el mismo Pedro quien presenta una lista de candi-

El (Pedro) presentó ${ }^{49}$ a dos, a José, a quien llaman Bernabe $e^{50}$ y que tenía el sobrenombre de Justo, y a Matías ${ }^{51}$.

El primer sobrenombre del primer candidato, "José Bernabé», estaría activado en este momento ( $\tau o ̀ v ~ \kappa a \lambda o u ́ \mu \varepsilon v o v$, part. pr.: «a quien llaman» los apóstoles: ver 4,36); el segundo sobrenombre, "Justo", le habría sido asignado con anterioridad (ôs $\dot{\varepsilon} \pi \varepsilon \kappa \lambda \eta \dot{\theta \eta}$, aor. pas.); era la máxima calificación que se podía predicar de un judío. Según el Códice Beza, pues, Pedro se aseguraba, de un lado, que el candidato que encabezaba la lista gozase del beneplácito del grupo apostólico; por otro, que ya gozaba de la aprobación del mundo judío. Al último de la lista no le añade ningún calificativo: simplemente Matías. Astutamente ha pretendido inclinar el voto de los Ciento veinte a favor del candidato de los Apóstoles. Matías era un desconocido (no figura en la onomástica de los Setenta).

Pedro habría activado aquí el sobrenombre de José ( B $\alpha \rho v \alpha \beta \tilde{\alpha} v$, part. pr.). Según lo que nos dirá más adelante, sin embargo, aparecerá desactivado el sobrenombre que le habían puesto los apóstoles en arameo (ó $\dot{\pi} \pi \kappa \lambda \eta-$

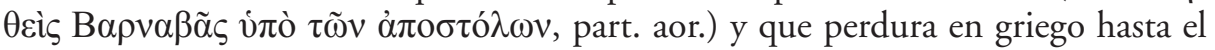

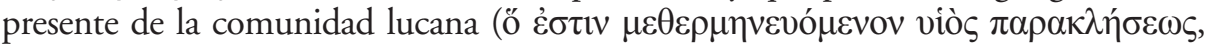
constr. perifrástica, 4,36).

Si seguimos, en cambio, el texto mayoritario, el primer candidato propuesto por los Ciento veinte sería "José Bersabé», un desconocido, al igual que Matías:

\footnotetext{
${ }^{48}$ Hch 1,21-22.

${ }^{49}$ ह̌ $\sigma \tau \sigma \varepsilon v \mathrm{D}^{*}$ gig; Aug.

${ }^{50}$ B $\alpha \rho v \alpha \beta \tilde{\alpha} \nu \mathrm{D} 1831^{\text {sup }} \mathrm{d} 6^{s}$ it vg ${ }^{\text {mss }}$ bo $^{\text {mss }}$; Or ${ }^{\text {lat }}$.

${ }^{51}$ Hch 1,33 D.
} 
Ellos presentaron ${ }^{52}$ a dos, a José, a quien llaman Bersabé $e^{53}$ y que tenía el sobrenombre de Justo, y a Matías.

\section{EL RESULTADO DE LA VOTACIÓN NO FAVORECIÓ AL CANDIDATO APOSTÓLICO}

A pesar de haber puesto Pedro los máximos calificativos sobre el candidato apostólico, el escrutinio de la votación nominal de los Ciento veinte no le favoreció:

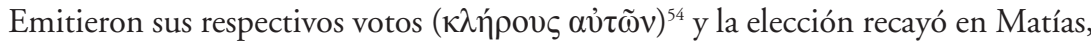
y fue contado ${ }^{55}$ con los Doce ${ }^{56}$ apóstoles $^{57}$.

Pedro, a pesar del voto de castigo que ha recibido su propuesta, ha evitado con esta estratagema que los hermanos de Jesús pudiesen hacer valer sus derechos de sangre. La restauración que los Once habían exigido a Jesús y que este había dejado truncada, ha sido ratificada por la asamblea de los Ciento veinte, si bien a precario: ¡Han restaurado para el reino de Israel... los Doce apóstoles! Según el Códice Beza, el candidato que ha resultado elegido no llenará en absoluto la vacante que había dejado Judas, sino tan solo ejercerá su función de administrador de la bolsa comunitaria. Aunque haya sido a precario, "fue contado con», la asunción de Matías en el grupo apostólico les permitirá presentarse ante Israel en representación de las doce tribus, tal como Jesús les había asignado al principio, "los Doce apóstoles». Según la mayoría de manuscritos, en cambio, puesto que hablan de una cooptación en toda regla ${ }^{58}$, «fue cooptado con/a los Once apóstoles», restablecen en la práctica el número Doce (Once + Matías), tal como habían pretendido que hiciese Jesús y este se había negado en absoluto a restaurarlo.

${ }^{52} \tilde{\varepsilon} \sigma \tau \eta \sigma \alpha \nu$ txt B $\aleph$ A rell.

${ }^{53}$ B $\alpha \rho \sigma \alpha \beta \beta \tilde{\alpha} v$ txt B $\mathrm{N}$ A rell; Bas Chrys Eus Oratrms Pap. El premeditado cambio de nombre impide asociar el candidato propuesto con el candidato apostólico (Hch 4,36) y con el profeta elegido por el Espíritu Santo, junto con el maestro Saulo, para que llevaran a cabo la misión a los paganos (Hch 13,2).

${ }^{54} \alpha \dot{\tau} \tilde{\omega} v \operatorname{txt}(\mathrm{ECM}) \mathrm{D}^{*} \mathrm{E} \Psi$ 61. 94. 180. 181. 307.323. 429. 431. 436. 453. 467. 522. 610. 614. 619.621.629. 630. 636. 966. 1162. 1241. 1270.1292. 1297. 1490.1501. 1505. 1595. 1611. 1678. 1751. 1831'. 1836. 1842. 1884. 2147. 2200. 2412. 2496. 2652. 2718. 2805 vg $^{\text {ms }}$ sy $^{\text {ht }}$ Bas Chrys I

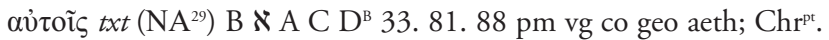

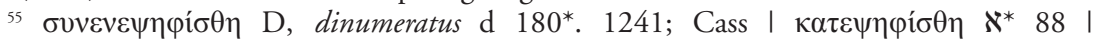

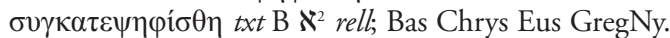

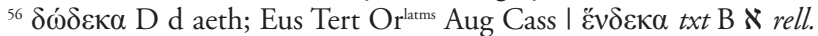

${ }^{57}$ Hch 1,26 .

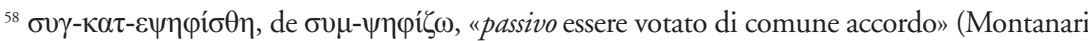

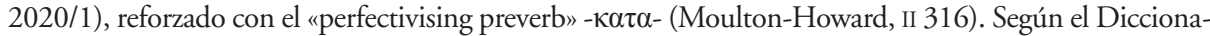
rio de la Real Academia Española, «cooptar. Llenar las vacantes que se producen en el seno de una corporación mediante el voto de los integrantes de ella». 
En conclusión, el hecho de no haber tenido en cuenta la preposición cí por suponer que se trataba de una duplografía del copista, nos ha privado de una información trascendental de cara a comprender la visión que tenían las comunidades que utilizaban el Códice Beza en lo que respecta al cambio de planes de Jesús momentos antes de la ascensión. En una edición crítica, las suposiciones de los editores son inaceptables.

RECIBIDO: diciembre 2019; ACEPTADO: enero 2020. 